\title{
Aeromonas spp as a potential cause of diarrhoea in children
}

M H Wilcox, A M Cook, A Eley, R C Spencer

\begin{abstract}
Aims: To determine the prevalence of Aeromonas spp in the faeces of children and the association with symptoms of gastroenteritis.
\end{abstract}

Methods: Faecal specimens $(n=1026)$ were cultured for Aeromonas spp using three selective media and an enrichment broth at both $30^{\circ} \mathrm{C}$ and $37^{\circ} \mathrm{C}$. The isolation of Aeromonas spp was correlated with symptoms of gastroenteritis, previous antibiotic use, and environmental temperature.

Results: Aeromonas spp $(\mathrm{n}=28)$ from 26 (2.5\%) patients were recovered. Bile salt, Irgasan, and brilliant green agar was the most efficient selective culture medium. Eleven of the patients had symptoms of gastroenteritis, usually mild diarrhoea of two to three days' duration, in the absence of other recognised enteropathogens. $A$ caviae was a particularly frequent isolate (nine out of 11 cases) in symptomatic individuals. Only one out of seven Aeromonas spp recovered by enrichment culture alone was possibly associated with symptoms of gastroenteritis. There was a close correlation between the environmental temperature and isolation of Aeromonas spp.

Conclusions: Aeromonas spp and particularly $\boldsymbol{A}$ caviae may cause gastroenteritis in children, most often during warmer months of the year. Culture for these potential enteropathogens could be confined to summer and autumn months. Bile salt, Irgasan, and brilliant green selective agar, but not alkaline peptone water enrichment, is an efficient culture medium for recovering possibly clinically important isolates.

(F Clin Pathol 1992;45:959-963)

Department of Experimental and Clinical Microbiology, The University of Sheffield Medical School, Beech Hill Road, Sheffield S10 2RX M H Wilcox

A M Cook A Eley R C Spencer Correspondence to: M H Wilcox

Accepted for publication 6 May 1992 dren, adults, community and hospital patients, for example) makes comparison of these rates difficult. Furthermore, as the detection of Aeromonas spp has been shown to be seasonally associated, ${ }^{256}$ studies should ideally report isolation rates over 12 month periods, or multiples thereof.

Of the three commonly recognised phenotypes, $A$ hydrophila and $A$ sobria have generally been considered to be the most virulent, with $A$ caviae rarely being implicated as a pathogen..$^{7-9}$ However, recent studies have indicated that $A$ caviae may indeed be an enteropathogen, particularly in children. ${ }^{51011}$ One of these $e^{11}$ noted an association between high stool $\mathrm{pH}$ and the isolation of $A$ caviae, possibly related to the poor tolerance (suicide phenomenon) of acidic conditions by this species. ${ }^{12}$

We report the results of a hospital-based study lasting 12 months to determine the isolation rate of Aeromonas spp from the faeces of children in Sheffield. Clinical evidence of enteropathogenicity for each of the major species was determined by recording symptoms and signs of gastroenteritis. Evidence to support the association between stool $\mathrm{pH}$ and A caviae isolation was also sought. To maximise the isolation rate and to determine the most efficient means of detecting Aeromonas spp in faeces, we used three different selective media at both $30^{\circ} \mathrm{C}$ and $37^{\circ} \mathrm{C}$, and also an enrichment medium.

\section{Methods}

A total of 1026 faecal specimens from children with gastrointestinal symptoms, submitted to the Bacteriology Department at the Sheffield Children's Hospital between November 1990 and October 1991, were examined. This represented $60 \%$ of all the faecal specimens received, as only those samples that could be processed within 24 hours of receipt were included in the study. Eighty five per cent of the specimens were from inpatients with the remainder equally divided between attenders at the Accident and Emergency Department and outpatients.

Routine investigations for enteropathogens were performed on all the study specimens. These included wet film microscopy, a modified Ziehl-Neelsen stain for cryptosporidia, culture on MacConkey's, deoxycholate, brilliant green and charcoal-cefaperazone agars, and selenite broth enrichment. Specimens were also examined by electron microscopy if virological investigation was requested by the clinician; $40 \%$ of the study specimens were ted in the faeces of controls in the same studies in $1 \%, 0 \%, 0 \%, 9 \%$, and $0 \%$ of cases. However, the use of varied isolation procedures and different study populations (chil- 


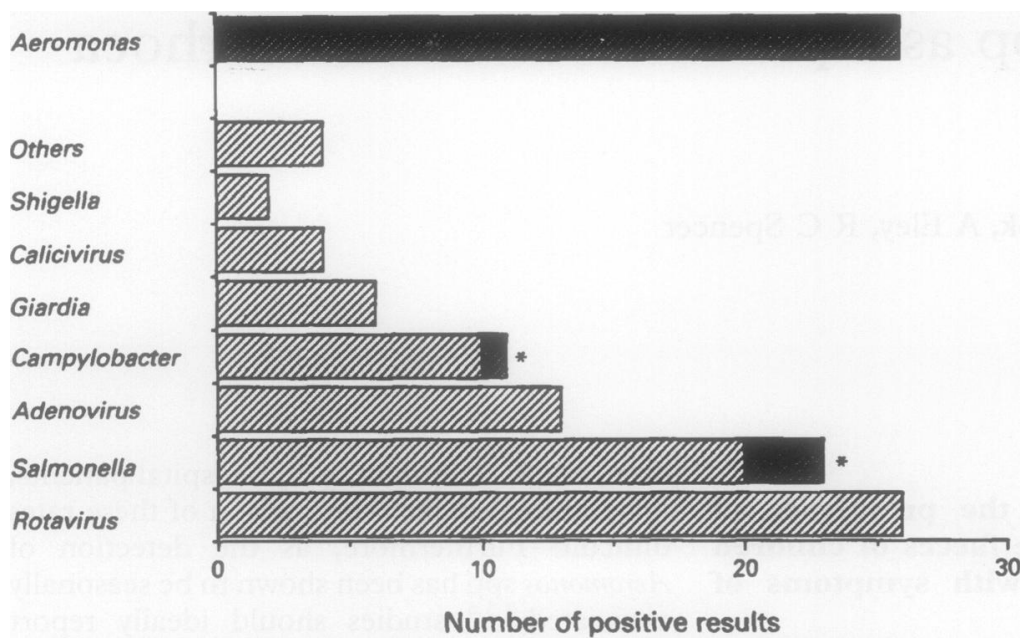

Figure 1 Enteropathogens and aeromonads isolated from 1026 faecal specimens. *Aeromonas isolated together with one campylobacter and three salmonellae. examined as such.

For the isolation of Aeromonas spp a sterile swab was used to inoculate faeces on to two whole plates of each of the following media: ampicillin (30 $\left.\mathrm{g} \mathrm{l}^{-1}\right)$ blood agar, comprising $7 \%$ horse blood and Oxoid Columbia agar base (ABA) ${ }^{13}$; Difco Aeromonas agar (bile salt, Irgasan, brilliant green agar) (DAA) ${ }^{14}$; and xylose deoxycholate agar (XDCA) (Life Technologies Ltd, Scotland). ${ }^{15}$. One of each pair of plates was incubated overnight at $30^{\circ} \mathrm{C}$ and the other at $37^{\circ} \mathrm{C}$. A loopful of faeces was also inoculated into alkaline peptone water (pH8.6), which was incubated overnight at $37^{\circ} \mathrm{C}$ and then subcultured on to half-plates of ABA, DAA, and XDCA.

After incubation, potential Aeromonas colonies (translucent colonies on DAA and pale yellow colonies on XDCA) were screened for oxidase production using filter paper impregnated with tetramethyl-p-phenylenediamine. All oxidase positive colonies were subcultured for purity, and a Hugh and Leifson (oxidation/ fermentation) test was done. Fermentative, oxidase positive isolates were tested for resistance to the vibriostatic agent 0129 by disc diffusion using $10 \mu \mathrm{g}$ and $150 \mu \mathrm{g}$ discs (Oxoid). Those isolates resistant to the higher level of 0129 were then phenospeciated by three methods: conventional biochemistry according to the criteria of Janda et al $^{16}$; the suicide phenomenon and aesculin production $^{12}$; API $20 \mathrm{NE}$ strips and haemolysin production (Wilcox MH, Cook AM, Geary I, Spencer RC, abstract 729; 17th International Congress of Chemotherapy 1991).

The $\mathrm{pH}$ of each study specimen was measured by direct contact between Whatman $\mathrm{pH}$ paper (BDH) and faeces.

Maximum and minimum temperatures for each day of the study period were obtained from the local meteorological office. This is situated immediately opposite the Sheffield Children's Hospital. The data were converted to mean weekly temperatures by averaging 14 daily figures.

Patients' details, including an antibiotic history, were collected to determine the potential significance of specimens positive for Aeromo- nas spp. The former data were collated without knowledge of the culture results. Diarrhoea was defined as multiple loose stools of more than one day's duration. Gastroenteritis was considered to be present if there was diarrhoea plus or minus additional symptoms such as nausea, vomiting, and abdominal pain.

The $\chi^{2}$ test with Yates' correction for small numbers was used to compare the numbers of $A$ caviae in symptomatic and asymptomatic patients; the Wilcoxon rank sum test was used to compare their ages.

\section{Results}

A total of 89 accepted enteropathogens were recovered from faecal specimens, equivalent to an isolation rate of $8.7 \%$ (fig 1 ). Twenty six specimens $(2 \cdot 5 \%)$ contained 28 Aeromonas spp isolates (two each containing two different isolates). In four of these 26 cases Aeromonas spp were isolated together with known enteropathogens (three salmonellae and one campylobacter). The 28 Aeromonas spp comprised 17
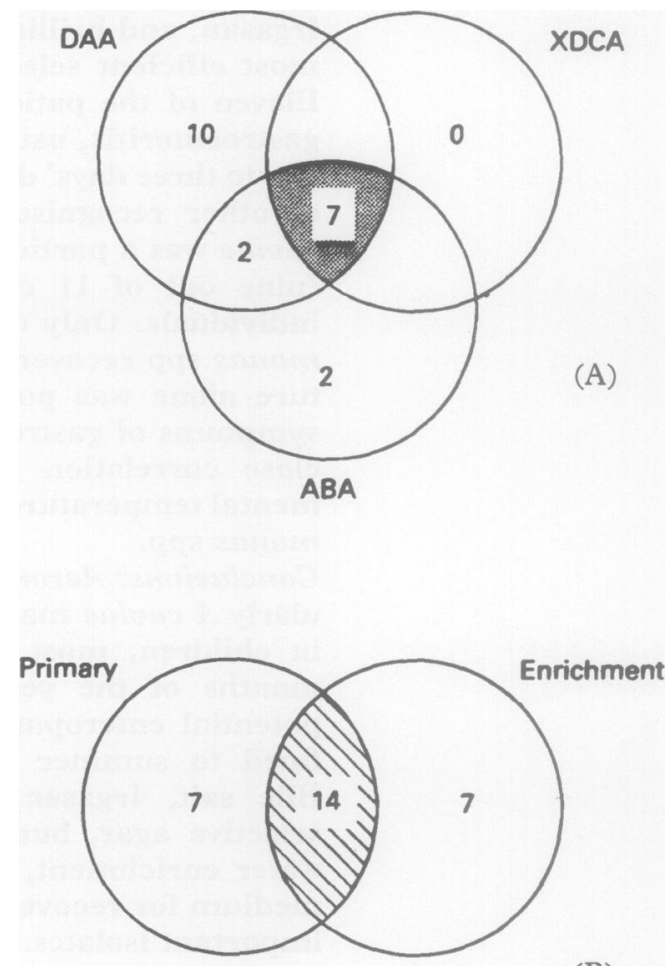

(B)

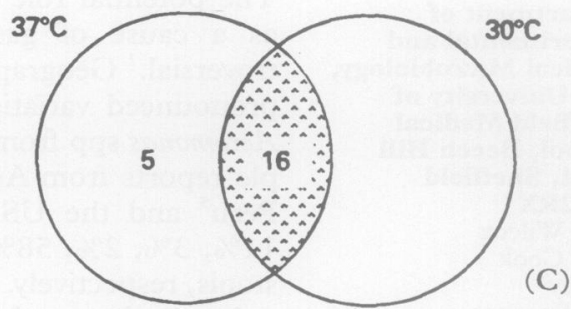

Figure 2 Isolation of aeromonads according to primary media type $(A)$, primary or enrichment culture $(B)$, and temperature of primary culture $(C)$. Figures refer to number of isolates. 


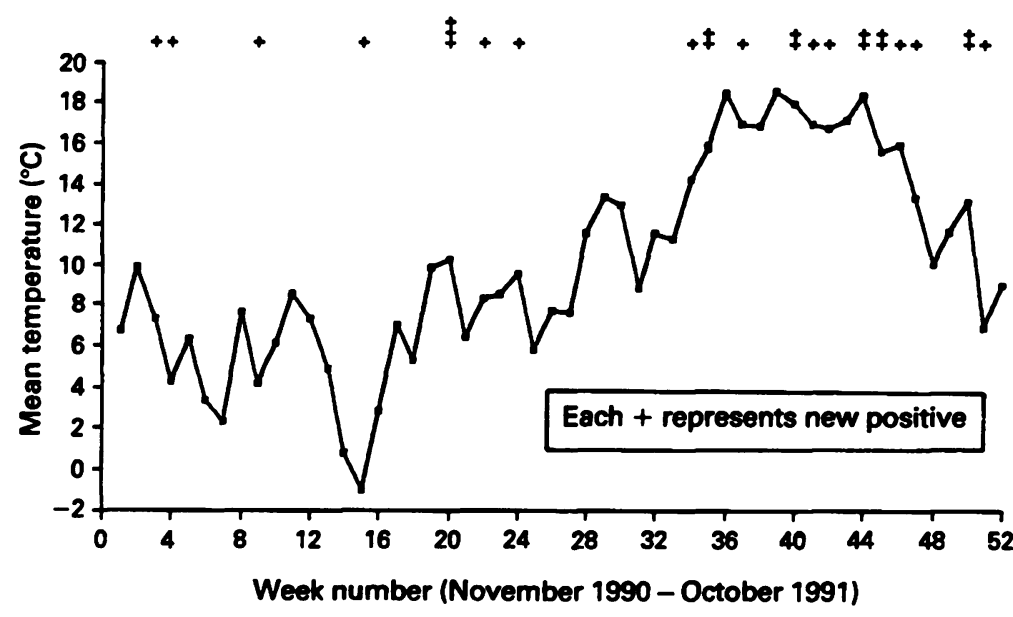

Figure 3 Correlation between mean weekly atmospheric temperature over 12 months and isolation of Aeromonas spp from faeces.

(61\%) $A$ caviae, eight (29\%) $A$ hydrophila, two (7\%) $A$ sobria, and one isolate that could not be identified. Apart from the latter isolate, the three different methods of phenospeciation gave concordant results.

Figure 2 shows the relation between culture media and temperature conditions with the isolation of Aeromonas spp. Of the 21 isolates recovered on primary culture plates, 19 grew on DAA, 11 on ABA, and seven on XDCA. All the isolates grew on either DAA or ABA, although a quarter were recovered only after alkaline peptone water enrichment. Notably, only two of the seven isolates recovered by enrichment alone came from patients with symptoms of gastroenteritis (see below); in one of these cases an accepted enteropathogen was also isolated. Incubation of primary cultures at $37^{\circ} \mathrm{C}$ increased the yield of positive results by five isolates compared to $30^{\circ} \mathrm{C}$. The routine laboratory methods failed to detect Aeromonas spp in any of the study faecal specimens.

Interestingly, of the 28 isolates, 17 were later found to be lactose positive on MacConkey's agar; only three out of $17 \mathrm{~A}$ caviae isolates were lactose negative. The $\mathrm{pHs}$ of faeces from which $A$ caviae was isolated (range $6 \cdot 0-8 \cdot 5$, median $7 \cdot 0$ ) were similar to those containing other Aeromonas spp (6.0-8.5, median 7.0), and also to negative specimens $(6 \cdot 0-8 \cdot 5$, median $6 \cdot 5)$.

The isolation of Aeromonas spp was more common during periods of warmer weather

Relation of Aeromonas spp isolated to clinical setting

\begin{tabular}{|c|c|c|c|}
\hline Clinical setting & $\begin{array}{l}\text { No of cases positive } \\
\text { for Aeromonas spp }\end{array}$ & $\begin{array}{l}\text { Antibiotic use within } \\
\text { previous two weeks }\end{array}$ & $\begin{array}{l}\text { Identity of Aeromonas } \\
\text { spp }\end{array}$ \\
\hline $\begin{array}{l}\text { Diarrhoea (no } \\
\text { accepted } \\
\text { enteropathogen) }\end{array}$ & 11 & 3 & $\begin{array}{l}9 A \text { caviae } \\
2 A \text { hydrophila }\end{array}$ \\
\hline $\begin{array}{l}\text { Diarrhoea (with } \\
\text { accepted } \\
\text { enteropathogen) }\end{array}$ & 4 & 0 & $\begin{array}{l}3 \text { A caviae } \\
1 \text { A sobria }\end{array}$ \\
\hline No diarrhoea & $11^{\star}$ & 6 & $\begin{array}{l}5 A \text { caviae } \\
6 A \text { hydrophila } \\
1 A \text { sobria } \\
1 \text { Unknown }\end{array}$ \\
\hline
\end{tabular}

* In two cases both $A$ caviae and $A$ hydrophila were isolated; one of the isolates could not be speciated. (fig 3). There were only two positive faecal specimens when the mean weekly environmental temperature was less than $7^{\circ} \mathrm{C}$. After a cluster of isolations in March-April 1991 (weeks 20-24), two months (weeks 25-33) elapsed without any positive specimens. In March 1991 (weeks 17-21) the mean temperature was $8^{\circ} \mathrm{C}$ compared with a 30 year (1951-1980) historical average of $5 \cdot 5^{\circ} \mathrm{C}$. Conversely, the month of June (weeks 30-34) saw a mean temperature of $12.2^{\circ} \mathrm{C}$ which was $2 \cdot 2^{\circ} \mathrm{C}$ lower than the historical average.

The age range (and median age) of all study patients and of those with Aeromonas spp in their faeces was 1 week to 15 years (11 months) and 1 month to 9 years (16 months), respectively. Symptomatic Aeromonas positive patients were generally younger (1 month-3 years; median 1 year) than asymptomatic individuals ( 5 months- 9 years; median 2 years); $0.05<p<0.1$. Of the 26 patients from whom Aeromonas spp were isolated, 11 had no diarrhoea nor any other symptoms of gasteroenteritis (table 1). Six of them, however, were either receiving antibiotics at the time of isolation or had done so within the previous two weeks; three patients received amoxycillin, two had penicillin and flucloxacillin, and one was given cefuroxime and metronidazole. Faecal specimens were sent to the laboratory in five of these 11 cases because of the passage of a single loose/offensive stool. Three of the 11 Aeromonas positive patients who did have diarrhoea (but no accepted enteropathogen) had a recent history of antibiotic treatmentone with amoxycillin, one with penicillin, and one with erythromycin. The duration of diarrhoea ranged from two to 21 days, with a median of three days. Two patients had associated abdominal pain and three had vomiting, of which one (a 15 month old baby with an ileostomy) became clinically dehydrated. Although the numbers are small, the isolation of $A$ caviae was particularly common in symptomatic (nine out of 11) compared with asymptomatic patients (five out of 11); $\mathrm{p}>0 \cdot 1$.

\section{Discussion}

Accumulating evidence suggests that in at least a proportion of cases Aeromonas spp can cause gastroenteritis. ${ }^{25}$ There are several weaknesses in this hypothesis: a lack of a suitable animal model; inconsistent results in human volunteer challenge studies ${ }^{17}$; and an absence of reported outbreaks of infection. As the major virulence determinants of Aeromonas spp have still to be clarified, it is difficult to perform adequate animal and human challenge studies. Strains used in such work may lose their pathogenicity during subculture and storage. A recent report documented for the first time that an identical strain of $A$ hydrophila was isolated from a patient with acute gastroenteritis and from recently consumed prawns. ${ }^{18}$

This study is the second largest United Kingdom report of the prevalence of Aeromonas spp in the faeces of paediatric inpatients. 
An earlier report noted that $4.4 \%$ of specimens contained Aeromonas spp, but did not correlate isolation with clinical details. The isolation rate of Aeromonas spp from routine faecal specimens in this study was $2.5 \%$, similar to that for the accepted enteropathogens salmonellae $(2 \cdot 2 \%)$ and rotavirus $(2 \cdot 5 \%)$. We considered the possibility of collecting specimens from a control population of asymptomatic children, but following earlier unsuccessful results in a pilot study this proved impracticable. Our use of three culture media, an enrichment broth, and two culture temperatures to isolate Aeromonas spp makes it unlikely that we had false negative specimens. Several different commercially available selective media and ampicillin blood agar have been suggested for isolating Aeromonas spp. ${ }^{120}$ In our hands DAA performed very well, and in only two out of 21 cases did Aeromonas spp fail to grow on it on primary culture; these two isolates were recovered on ABA. DAA contains brilliant green, bile salts, and the disinfectant Irgasan as selective agents. The usefulness of brilliant green has been noted earlier, ${ }^{21}$ and Irgasan has also been incorporated successfully into another selective medium (CIN; cefsulodin, irgasan, novobiocin). ${ }^{20}$ DCA inhibits commensal flora by virtue of bile salts, but it performed poorly in the present study and in this formulation cannot be recommended.

We confirmed the findings of others that the use of alkaline peptone water enrichment increases the yield of faecal specimens positive for Aeromonas spp. ${ }^{21} 22$ Studies that do not incorporate such enrichment techniques are likely to underestimate significantly the prevalence of Aeromonas spp in faeces. However, the clinical importance of isolates recovered by enrichment alone must be considered to be extremely doubtful. Robinson et al found that the use of enrichment culture frequently detected Aeromonas spp in asymptomatic individuals. ${ }^{23}$ Similarly, we observed that only one out of seven isolates detected by alkaline peptone water enrichment alone came from a patient with gastroenteritis, in the absence of a recognised pathogen.

We were prompted to compare isolation at $30^{\circ} \mathrm{C}$ and $37^{\circ} \mathrm{C}$, because preliminary studies with a collection of 30 Aeromonas spp had shown two strains that failed to grow on at least one of the media at $37^{\circ} \mathrm{C}$. However, no isolates were recovered from faeces on media incubated at $30^{\circ} \mathrm{C}$ compared with those incubated at $37^{\circ} \mathrm{C}$.

Clinical assessment of Aeromonas spp positive individuals not otherwise infected with a known enteropathogen suggested that half of the cases had symptoms attributable to these micro-organisms. Undoubtedly, Aeromonas spp are carried asymptomatically by some individuals. ${ }^{524}$ However, this is also true for well recognised enteropathogens such as salmonellae. In this study children with gastroenteritis and Aeromonas spp in their faeces generally had non-profuse diarrhoea of short duration (median three days). Vomiting and abdominal pain were present in only a few cases. The affected children were 3 years old or younger, while four (36\%) of those carrying Aeromonas spp asymptomatically were at least 5 years old. An association between antibiotic use and isolation of Aeromonas spp noted elsewhere was most apparent in asymptomatic children in the present study. ${ }^{25} A$ caviae predominated among the clinical isolates, particularly in those from symptomatic individuals $(82 \%)$. These results support the recent findings that $A$ caviae seems to be a relatively common isolate from the faeces of young children with gastroenteritis. ${ }^{51011} \mathrm{We}$ were unable to confirm an association between alkaline faecal $\mathrm{pH}$ and isolation of $A$ caviae. ${ }^{11}$ However, the patients positive for $A$ caviae reported by Namdari and Bottone ${ }^{11}$ were mostly less than 1 year old. In our series the three infants of less than 1 year old with gastroenteritis possibly caused by $A$ caviae had stool pHs of 8.0 (twice) and 7.5; all were bottle fed. The alkaline faecal $\mathrm{pH}$ found in bottle fed babies may indeed help to select for $A$ caviae and explain the relatively high isolation rate of this species in infants.

Aeromonas spp are widely distributed in fresh water, sewage, marine environments close to freshwater outlets, and drinking water. ${ }^{26-28}$ Maximal levels of these bacteria in fresh water occur during summer and early autumn. Aeromonas spp have also been suggested as potential foodborne pathogens (see above), which is supported by their high prevalence in supermarket meat samples (58-74\%) and raw milk $(27 \%) .^{29}$ Our data confirm the close relation between environmental temperature and isolation of Aeromonas spp from faeces. ${ }^{5625}$ Laboratories could focus their efforts to isolate these bacteria during the warmer months to limit the additional costs of the extra media required.

We would encourage attempts to isolate Aeromonas spp from well defined populations to determine more closely their prevalence and potential to cause gastroenteritis. Selective (DAA) but not enrichment media are recommended. Uncertainties concerning the taxonomy of Aeromonas spp and the virulence of given species will not be resolved without such studies.

We thank Difco Laboratories Ltd and Life Technologies Ltd for kindly supplying their respective selective media for use in this study.

1 Wadstrom T, Ljungh A. Aeromonas and Plesiomonas as food- and waterborne pathogens. Int $\mathcal{f}$ Food Microbiol 1991;12:303-12.

2 Burke V, Gracey M, Robinson J, Peck D, Beaman J, Bundell C. The microbiology of childhood gastroenteritis: Aeromonas species and other infective agents. $\mathcal{F}$ Infect $D$ is 1983;148:68-74.

3 Mikhail IA, Fox E, Haberberger RL, Ahmed MH, Abbatte EA. Epidemiology of bacterial pathogens associated with infectious diarrhoea in Diibouti. $\mathcal{f}$ Clin Microbiol 1990 28:956-61.

4 Deodhar LP, Saraswathi K, Varudkar A. Aeromonas spp and their association with human diarrheal disease. $\mathcal{F}$ Clin Microbiol 1991;29:853-6.

5 Pazzaglia G, Sack RB, Salazar E, et al. High frequency of coinfecting enteropathogens in Aeromonas-associated coinfecting enteropathogens in Aeromonas-associated 1991;29:1151-6.

6 Agger W A, McCormick J D, Gurwith M J. Clinical and microbiological features of Aeromonas hydrophila-assomicrobiological features of Aeromonas hydrophil
ciated diarrhea. $\mathcal{f}$ Clin Microbiol 1985;21:909-13.

7 Millership SE, Barer MR, Tabaqchali S. Toxin production by Aeromonas spp from different sources. $\mathcal{F}$ Med Microbiol 1986;22:311-14

8 Watson I M, Robinson JO, Burke V, Gracey M. Invasiveness of Aeromonas spp in relation to biotype, virulence factors, 
and clinical features. $f$ Clin Microbiol 1985;22:48-51.

9 Janda JM, Clark RB, Brenden R. Virulence of Aeromonas species as assessed through mouse lethality studies. Cur Microbiol 1985;12:163-8.

10 Namdari H, Bottone EJ. Cytotoxin and enterotoxin production as factors delineating enteropathogenicity of Aeromonas caviae. F Clin Microbiol 1990;28:1796-8.

11 Namdari H, Bottone EJ. Microbiologic and clinical evidence supporting the role of Aeromonas caviae as a dence supporting the role of Aeromonas caviae as
pediatric pathogen. $\mathcal{f}$ Clin Microbiol 1990;28:837-40.

12 Namdari H, Bottone EJ. Suicide phenomenon in mesophilic aeromonads as a basis for species identification. $\mathcal{f}$

13 George WL, Nakata MM, Thompson J, White ML. Aeromonas-related diarrhoea in adults. Arch Intern Med 1985;145:2207-11.

14 Hunt GH, Price EH, Patel U, Messenger L, Stow P, Salte P. Isolation of Aeromonas spp from faecal specimens. $\mathcal{F}$ Clin Pathol 1987;40:1382-91.

15 Shread P, Donovan TJ, Lee JV. A survey of the incidence of Aeromonas in human faeces. Society for Microbiology Quarterly 1981;8:184.

16 Janda MJ, Reitano M, Bottone EJ. Biotyping of Aeromonas isolates as a correlate to delineating a species-associated isolates as a correlate to delineating a species-ass

17 Morgan DR, Johnson PC, DuPont HL, Satterwhite TK Wood LV. Lack of correlation between known virulence properties of Aeromonas hydrophila and enteropathogenicity for humans. Infect Immun 1985;50:62-5.

18 Altwegg M, Lucchini G M, Luthy-Hottenstein J, Rohrbach M. Aeromonas-associated gastroenteritis after consumption of contaminated shrimp. Eur 7 Clin Microbiol Infect Dis 1991;10:44-5.

19 Robinson J, Burke V, Worthy PJ, Beaman J, Wagener L. Media for isolation of Aeromonas spp. from faeces. $\mathcal{f} \mathrm{Med}$ Microbiol 1984;18:405-11.
20 Altorfer R, Altwegg M, Zollinger-lten J, von Graevenitz A. Growth of Aeromonas spp. on celsulodin-irgasan-novobiocin agar selective for Yersinia enterocolitica. $\mathcal{F}$ Clin Microbiol 1985;22:478-80.

21 Millership SE, Chattopadhyay B. Methods for the isolation of Aeromonas hydrophila and Plesiomonas shigelloides from faeces. $\mathcal{F} \mathrm{Hyg}$ (Camb) 1984;92:145-52.

22 von Gravenitz A, Bucher C. Evaluation of differential selective media for isolation of Aeromonas and Plesiomonas spp from human faeces. I Clin Microbiol 1983; nas spp

23 Robinson J, Beaman J, Wagener L, Burke V. Comparison of direct plating with the use of enrichment culture for isolation of Aeromonas spp. from faeces. $\mathcal{F}$ Med Microbiol 1986;22:315-7.

24 Pazzaglia G, Escalante JR, Sack PB, Rocca C, Benavides V. Transient intestinal colonisation by multiple phenotypes of Aeromonas species during the first week of life. $\mathcal{F}$ Clin Microbiol 1990;28:1842-6.

25 Moyer NP. Clinical significance of Aeromonas species isolated from patients with diarrhoea. $f$ Clin Microbiol 1987;25:2044-8.

26 Burke V, Robinson J, Gracey M, Peterson D, Partridge K Isolation of Aeromonas hydrophila from a metropolitan Isolation of Aeromonas hydrophila from a metropolitan
water supply: correlation with clinical isolates. Appl water supply: correlation with

27 Rippey SR, Cabelli VJ. Occurrence of Aeromonas hydrophila in limnetic environments: relationship of the organism to trophic state. Microbiol Ecol 1980;6:45-54

28 Monfort $P$, Baleux B. Distribution and survival of motile Aeromonas spp. in brackish water receiving sewage treatment effluent. Appl Environ Microbiol 1991;57: 2459-67.

29 Ibrahim A, Mac Rae IC. Incidence of Aeromonas and Listeria spp. in red meat and milk samples in Brisbane, Australia. Int $\mathcal{f}$ Food Microbiol 1991;12:263-70. 